\title{
A Truncated Waveguide Fed by a Microstrip as a Radiating Element for High-Performance Automotive Anticollision Radars
}

\author{
Giovanni Andrea Casula, Giuseppe Mazzarella, and Giorgio Montisci \\ Dipartimento di Ingegneria Elettrica ed Elettronica, Università di Cagliari, Piazza d’Armi, 09123 Cagliari, Italy \\ Correspondence should be addressed to Giuseppe Mazzarella, mazzarella@diee.unica.it
}

Received 13 August 2012; Revised 10 October 2012; Accepted 10 October 2012

Academic Editor: Zhongxiang Q. Shen

Copyright ( $) 2012$ Giovanni Andrea Casula et al. This is an open access article distributed under the Creative Commons Attribution License, which permits unrestricted use, distribution, and reproduction in any medium, provided the original work is properly cited.

\begin{abstract}
A small truncated waveguide fed by a microstrip line through a transverse coupling slot is proposed and assessed as a highperformance antenna and array element in the $\mathrm{K}$ band and above. This antenna allows to obtain a high radiated power, with a very low cross-polar component in the radiated field. It is therefore particularly suitable for application in automotive anticollision radars. The proposed radiating element has been analyzed by a numerical code based on an in-house method of moments, and the microstrip feeding line has been modeled by its equivalent magnetic-wall waveguide. A linear array of such elements has been designed and matched with a BPF-inspired matching network allowing an in-band behavior suitable for anti-collision radar use, with an out-of-band rejection large enough to avoid the first receiving BPF.
\end{abstract}

\section{Introduction}

The research in automotive anticollision radars (AAR) started in the 60's [1-4]. This technology measures the velocity and angle of a target by using a short-wavelength highresolution millimeter waves radar, giving an automatic vision of the environment in which the vehicle is moving. This information allows drawing the data required to perform specific actions to guarantee, as much as possible, safety for the vehicle and its passengers. AAR systems require compact size, high-speed data transfer, low-cost, and short-distance radio links. These requirements can be easily fulfilled using millimeter waves, where the shorter wavelength allows the use of small antennas to achieve the required gain. In designing radar systems for automotive applications, it is necessary to comply with national constraints on the allowed frequencies and power levels. In particular, several recommendations have been already issued by the national and international agencies [5], establishing strict limits both on the power levels and the spectral occupancy.

Two kinds of radar can be used in automotive applications: Short Range Radars, with visibility range limited to $30 \mathrm{~m}$, and Long Range Radars, with visibility range up to $150 \mathrm{~m}$. In this work, we will focus on Short Range AAR. The typical operating frequency of Short Range AAR antennas is $24 \mathrm{GHz}$, namely, within the $\mathrm{K}$ band. Their antennas must be simple, easy to mount, slim enough to remain inside the automobile outline, and efficient.

Arrays of microstrip patch antennas are a common choice for AAR [6-10], in particular, in the millimeter wave band, both for their small weight and size, low production cost, and ease of fabrication and integration either on a single grounded slab [11] or using a triplate structure [12]. The main drawbacks of such array elements are the low efficiency, the narrow frequency bandwidth, the relatively high crosspolar component of the radiated field, and the propagation of the surface wave in the dielectric substrate, which causes power loss and coupling.

We propose here a different array element, shown in Figure 1. This antenna consists of a truncated rectangular waveguide radiator [13], which replaces the patch (and its substrate). This truncated waveguide is fed by the electromagnetic coupling produced by a microstrip transverse slot (Figure 1). The radiated power can be modulated by acting on various parameters, such as the length of the coupling slot, its width, and, to a certain extent, the truncated waveguide length. Moreover, a thin dielectric slab lying on 


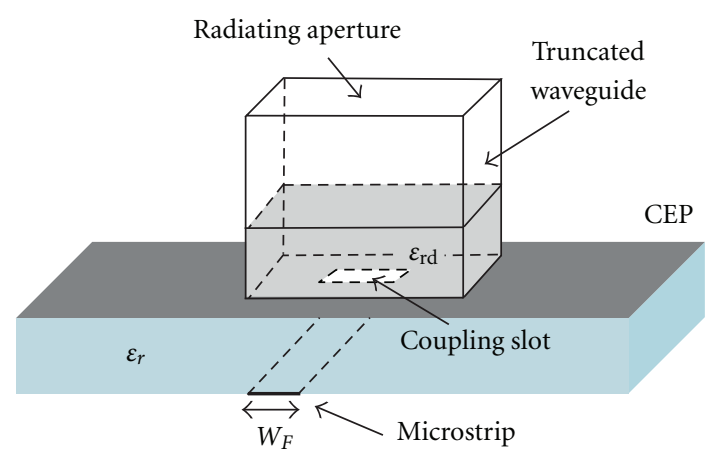

FIgURE 1: Radiating element geometry.

the slot can be placed inside the radiating element to increase the degrees of freedom in the design process.

This antenna allows higher efficiency, and a much lower cross-polar component and coupling level [13], with respect to a standard patch antenna. In fact, in the latter case, the mutual coupling is mainly due to the propagation of the surface wave in the dielectric substrate shared by the radiating patches, whereas the configuration proposed in this paper suppresses the surface wave in the radiating half-space. Furthermore, the use of a planar feeding structure allows to realize arrays with as much flexibility in the feeding network as standard printed triplate arrays.

The potential drawback of the proposed structure (shared by the triplate patch solution) could be the backradiation of the coupling slot. However, in our solution, the back-radiation due to the coupling slot is not high since the slot is not resonant (having typical dimensions equal or less than a quarter wavelength) and can be minimized by a suitable choice of the structure parameters, as we will show in Section 3.

The analysis of the antenna in Figure 1 has been performed by replacing the feeding microstrip line with its equivalent magnetic-wall waveguide (MWW) model [14, 15], and using the well-assessed method of moments (MoM) procedure developed by the authors in some previous works [13, 15-18].

The antenna of Figure 1 is well suited to be used as an array element. As a matter of fact, an array of truncated waveguides can be easily realized as a metallic plate with holes [19] and the feeding network requires a standard PCB technology. This realization is thicker than a simple PCB antenna but the total thickness is quite limited and well compensated by the increase of the electromagnetic performance.

To assess the use of the proposed antenna as an AAR array element, an 8-element linear array has been designed, with a broadside radiation and $-20 \mathrm{~dB}$ SLL, a bandwidth of $200 \mathrm{MHz}$ around the design frequency of $24 \mathrm{GHz}$, and matched. The resulting design has been verified using Ansys HFSS [20].

The radiation pattern is substantially within the specifications, thus assessing the element model. However, the input admittance is a strongly resonant one, so a matching network, derived from a band-pass filter model [21], has been employed. This allows to reach the required input bandwidth of $200 \mathrm{MHz}$, but we have also a very strong rejection out of the required bandwidth. Therefore, the radar receiving chain does not need an input BPF, with a significant reduction of complexity [22].

\section{Radiating Element Geometry and Behavior}

The proposed radiating element consists of a microstrip feeding line with a transverse coupling slot, cut in its ground plane, which feeds a truncated waveguide (Figure 2). The truncated waveguide can be partially filled with a dielectric slab lying on the slot. The slot between the apertures $\Sigma^{i}$ and $\Sigma^{e}$ radiates into the region bounded by the waveguide, and the aperture $\Sigma^{r}$ is the antenna element, which radiates into free space. In order to facilitate the realization, this truncated waveguide can be obtained as a hole in a thick metallic plate. Therefore, the actual radiating element used in this paper is shown in Figure 2(a). In Figures 2(b) and 2(c), the truncated waveguide ground plane is not reported for the sake of simplicity.

The analysis of the microstrip transverse slot and its interaction with the radiating aperture is performed using a full-wave technique, the method of moments in the Galerkin formulation $[16,23]$. The microstrip is modeled by a magnetic wall waveguide, as shown in [15]. Each aperture in Figure 2 is replaced by equivalent magnetic currents. Entire domain sinusoidal basis functions have been used in the regular apertures of the radiating element, thus allowing a very effective and accurate analysis [13]. The reader is referred to Section 2 of [13] for the details of the MoM procedure.

The geometrical parameters of the radiating element must fulfill the following requirements:

(a) the microstrip feeding line must be designed providing that, at the operating frequencies, only the fundamental TEM mode propagates;

(b) the transverse dimension $a$ of the truncated waveguide must be chosen in order that only the fundamental $\mathrm{TE}_{10}$ mode is excited, and the higher modes attenuation is large enough to prevent higher-order mode coupling between the slot and the radiating aperture;

(c) the transverse dimension $b$ must be smaller than the guided wavelength in the feeding microstrip line, which is the spacing between two elements in an array.

In the simulations presented in this section, the design frequency is $24 \mathrm{GHz}$ and the dielectric substrate is Roger Duroid 5800 with $\varepsilon_{r}=2.2$ and $h=0.381 \mathrm{~mm}$. In order to satisfy the previous requirements at the design frequency of $24 \mathrm{GHz}, a$ has been fixed to the value of $9.5 \mathrm{~mm}, b$ is equal to $4.75 \mathrm{~mm}$, and the width of the microstrip feeding line is $W_{F}=2 \mathrm{~mm}$, which corresponds to a characteristic impedance of $35 \Omega$, lower than the standard $50 \Omega$ to reduce back radiation. This choice allows to reach our purpose 


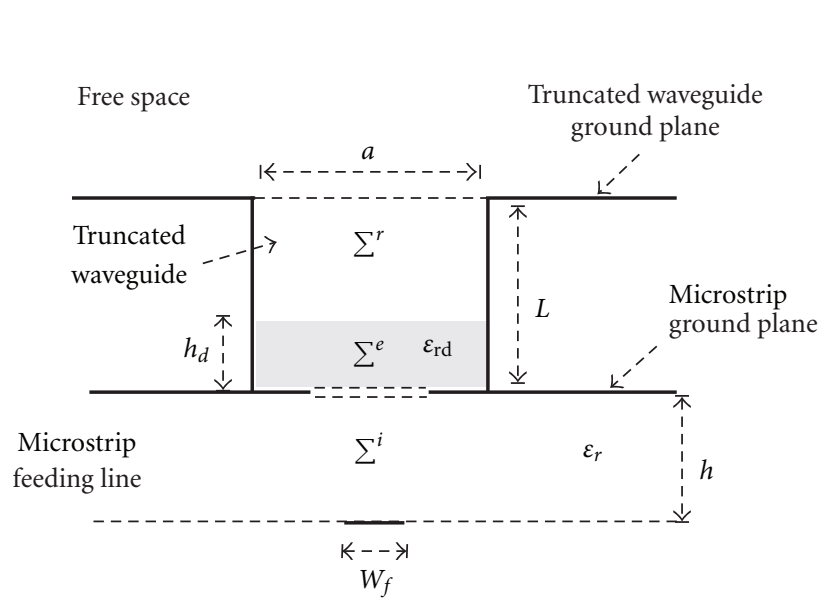

(a)

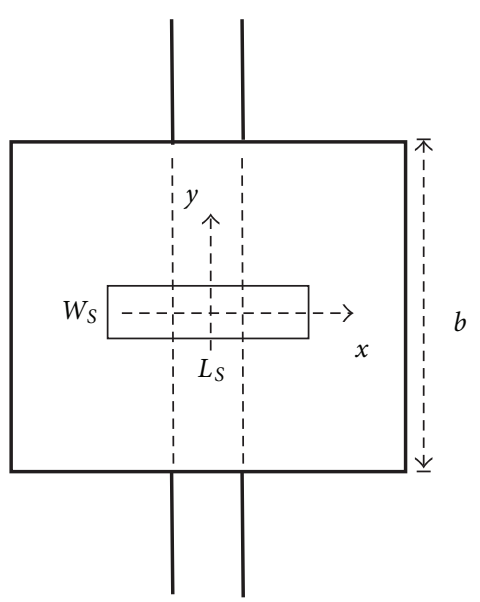

(b)

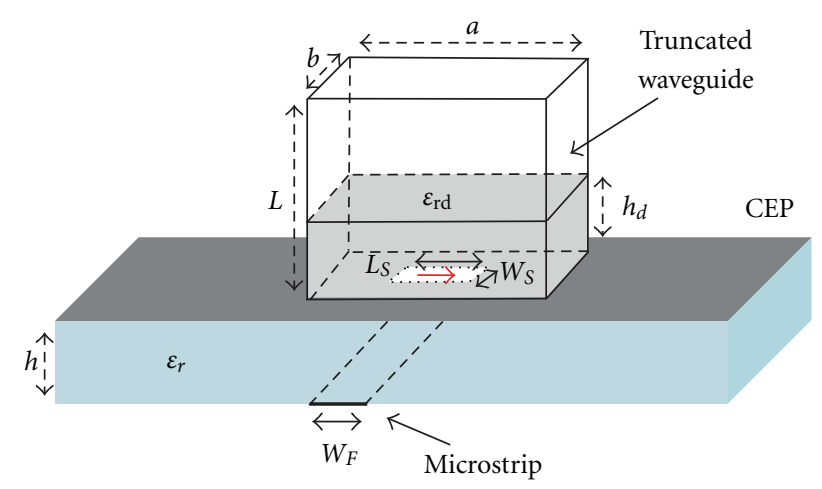

(c)

Figure 2: Radiating element geometry: (a) front view, (b) top view, and (c) 3D view.

with a simple realization and without modification of the main feeding line, though different and effective (but more expensive) solutions could be adopted (see e.g., $[24,25]$ ).

We have found that the equivalent circuit of the proposed antenna is a series impedance on the feeding microstrip line. Therefore, the antenna circuital properties can be described through its equivalent impedance. In Figure 3(a), the radiating element equivalent impedance, normalized to the microstrip feeding line impedance, is plotted.

The MoM procedure used here has been widely assessed in $[13,16-18]$. However, the characterization of the proposed radiating element needs also the validation of the MWW model for the microstrip line. Therefore, in Figure 3(a), we have performed a comparison between the structure fed by the microstrip line and the structure fed by its equivalent magnetic wall waveguide, both simulated with a general purpose FEM commercial software, Ansys HFSS. The results of our MoM procedure are also reported for a matter of completeness. These comparisons validate both the equivalence between the MWW and the microstrip for our application, and the MoM code. Though the results of the MoM code and HFSS are virtually equivalent, we would like to point out that, as reported also in [13], the computational time required by HFSS is about two orders of magnitude greater than the one required by our MoM code. Therefore, for the parametric analysis of the single radiating element we have utilized the MoM procedure.

Figure 3(b) shows the copolar radiated field in the $E$ plane and $\mathrm{H}$-plane at $24 \mathrm{GHz}$ both for the case of the antenna fed by a microstrip line and for the case of an equivalent MWW feeding. The cross-polar component is not shown since it is below $-40 \mathrm{~dB}$. The gain of this element is about $6.3 \mathrm{~dB}$. The difference in the radiated fields is due to the back radiation of the slot fed by the microstrip line, which is not considered in the case of the equivalent MWW. The back-radiation can be minimized by a suitable choice of the truncated waveguide parameters (namely, by maximizing the waveguide radiated power) and of the slot dimensions, as we will show in the next paragraph.

The circuital and radiating properties of the proposed antenna depend on the interaction between the aperture of the truncated waveguide and the coupling slot. In order to characterize the radiating element, we show its radiated power as a function of the slot length $L_{S}$ (Figure 4 ) and of the truncated waveguide length $L$ (Figure 5). Different values of the permittivity $\varepsilon_{\mathrm{rd}}$ of the dielectric substrate partially filling the truncated waveguide have been considered in both cases. The slot length variation shown in Figure 4 is superiorly limited by the effective length of the feeding microstrip line, since the equivalent MWW model for the microstrip line 


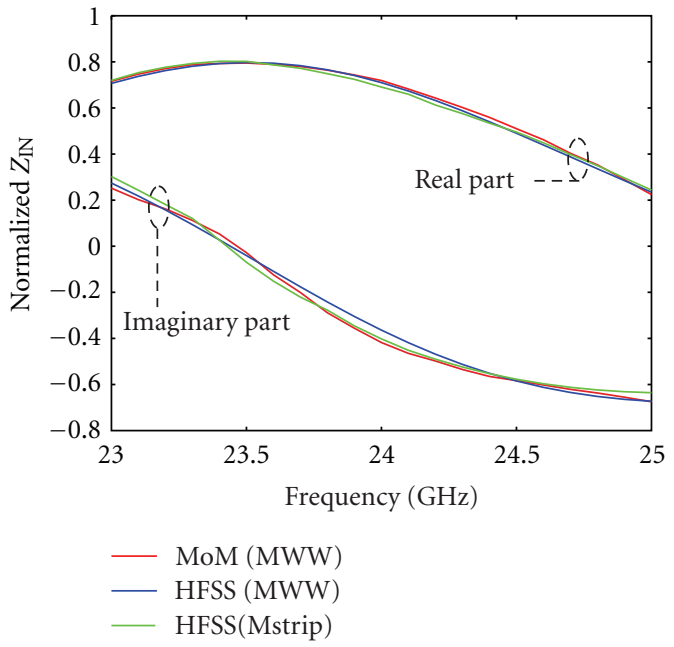

(a)

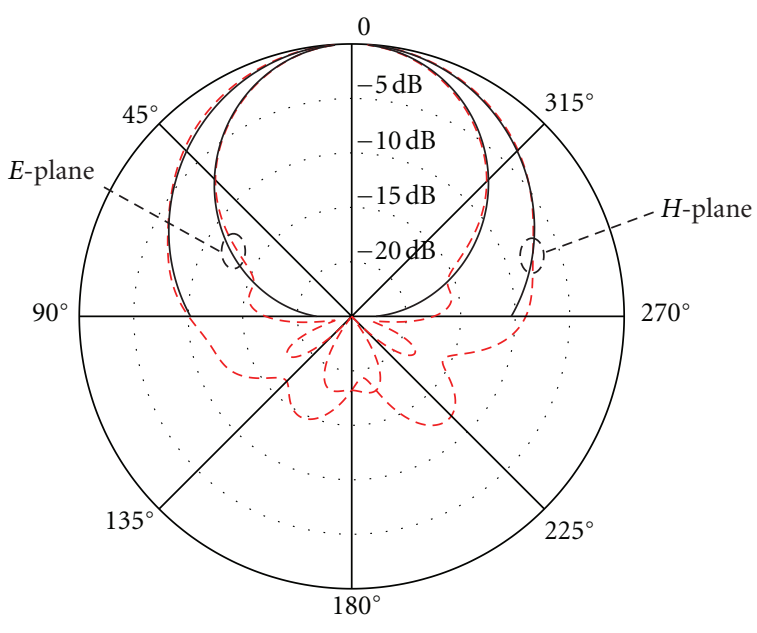

(b)

Figure 3: (a) Equivalent impedance; (b) far-field pattern. Continuous lines: equivalent MWW; dashed lines: microstrip. Radiating element parameters: $L_{S}=2.5 \mathrm{~mm}, W_{S}=0.25 \mathrm{~mm}$, and $L=10.8 \mathrm{~mm}$.

requires that the slot is completely inside the feeding MWW (this is not a strong limitation, since a transverse coupling slot is usually smaller than the MWW).

In Figure 5, we show the percentage of the radiated power as a function of the truncated waveguide length $L$. As apparent from these data, and as widely discussed also in [13], the radiated power, as a function of $L$, shows a periodicity of exactly $\lambda_{g t} / 2, \lambda_{g t}$ being the guided wavelength in the truncated waveguide. We can exploit this behavior, since it is not convenient using this radiating element for $L$ too small. Actually, in this case, the (cut-off) higher-order modes of the truncated waveguide (excited by the slot) are not attenuated enough at the waveguide radiating aperture, causing a degradation of the radiated field, and, therefore, of the antenna performance.

From the results shown in Figure 5, it is also apparent that by varying the truncated waveguide length, and with a suitable choice of the thickness and permittivity of the dielectric substrate inside the waveguide, we can modify the power radiated by the single element in a quite wide range.

\section{Array Design and Matching}

The radiating element presented in this paper has been used to design an 8-element linear array as automotive anticollision radar, with a broadside radiation and $-20 \mathrm{~dB}$ SLL. A rigorous design procedure for an array made with the proposed antenna is not available yet; therefore, the array has been designed neglecting the external mutual coupling between the truncated waveguides. We expect that this would affect only the input impedance [26], which can be easily matched, and not the radiation pattern.

We have selected the following parameters: $L=$ $10.36 \mathrm{~mm}, h=0.381 \mathrm{~mm}, \varepsilon_{r}=2.2, a=9.5 \mathrm{~mm}$ $b=4.75 \mathrm{~mm}$, and $W_{F}=2 \mathrm{~mm}$. Therefore, the only design parameters are the slot widths, $W_{S}$, and lengths, $L_{S}$. The element spacing must be equal to one wavelength in the feeding line and corresponds to $8.88 \mathrm{~mm}$ at the design frequency of $24 \mathrm{GHz}$ for the microstrip line and its equivalent MWW. The feeding microstrip line ends with a quarter wavelength termination beyond the last coupling slot.

In order to obtain a low back radiation, the radiated power of each element must be properly chosen. For the case of Figure 3, the maximum radiated power is about $5 \%$ (Figure 5), and the corresponding back-radiation is lower than $10 \mathrm{~dB}$ (Figure 3(b)). These are the typical values for an empty truncated waveguide. However, if the truncated waveguide is partially filled with a dielectric substrate lying on the slot (see Figures 1 and 2), the radiated power can be significantly increased, as shown in Figures 4 and 5, allowing to minimize the slot back-radiation.

Therefore, in Figure 6, we show the amplitude of the element excitation and the phase of the input impedance with respect to the slot width, for two different values of permittivity $\varepsilon_{\mathrm{rd}}$ of the dielectric layer inside the waveguide. The case of a truncated waveguide filled with air is also shown for comparison. The data from Figure 6 have been used to design the array. Actually, the choice of the array element design parameters, $L_{S}$ and $W_{S}$, must be performed minimizing the phase shift between the input impedances of the radiating elements, since the required excitations must be equiphase to get a broadside radiation. The best choice consists of fixing the slot lengths $L_{S}$ and choosing the slot widths $W_{S}$ which provide the desired excitations. In Table 1, we show the slot widths for the designed array and the corresponding elements excitations, obtained from Figure 6(a). Each radiating element has the same slot length $L_{S}=2 \mathrm{~mm}$, and all the truncated waveguides are partially 


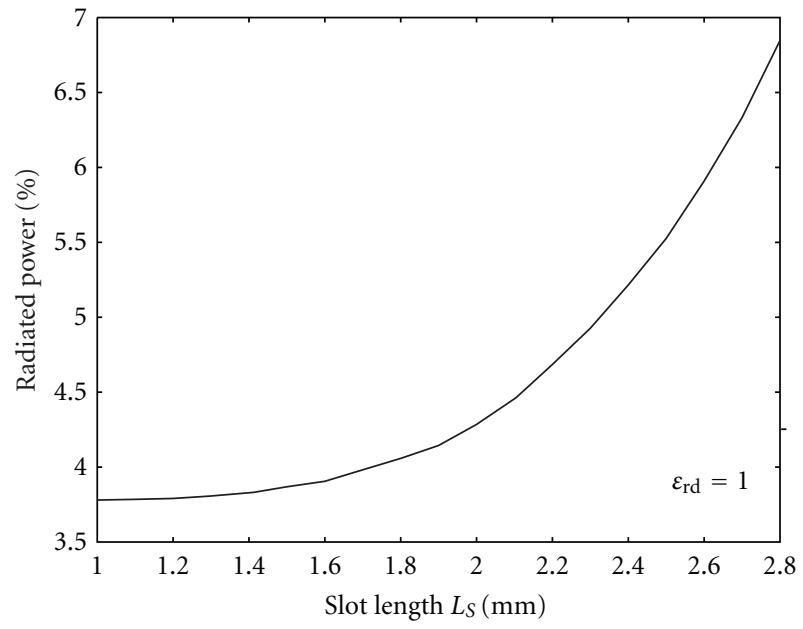

(a)

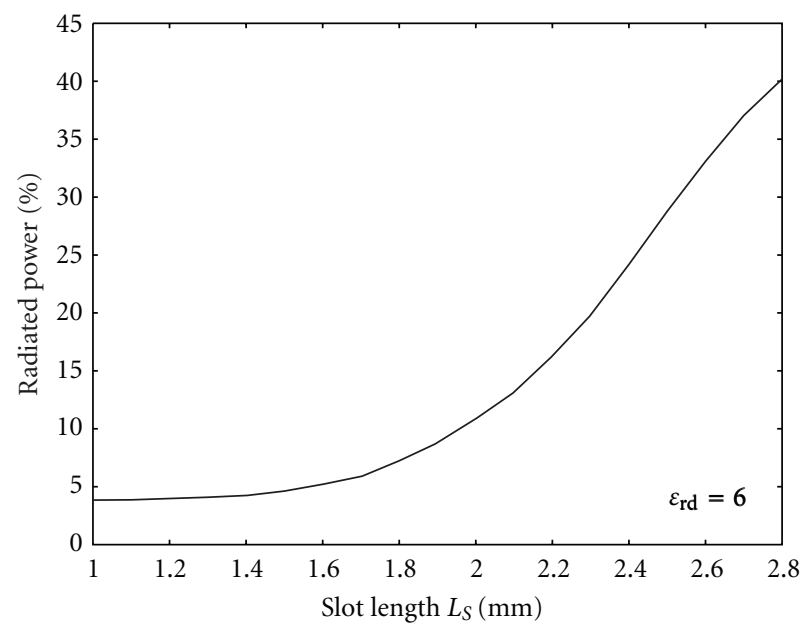

(b)

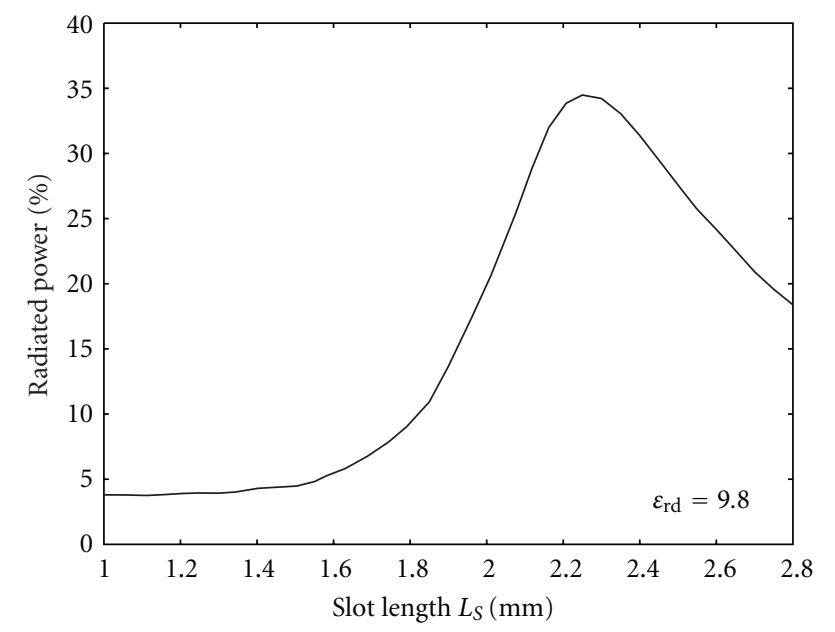

(c)

FIgURE 4: Radiated power (percentage of incident power) for the antenna in Figure 2. Radiating element parameters: $L=10.36 \mathrm{~mm}$, $W_{S}=0.3 \mathrm{~mm}$, and $h_{d}=1.2 \mathrm{~mm}$.

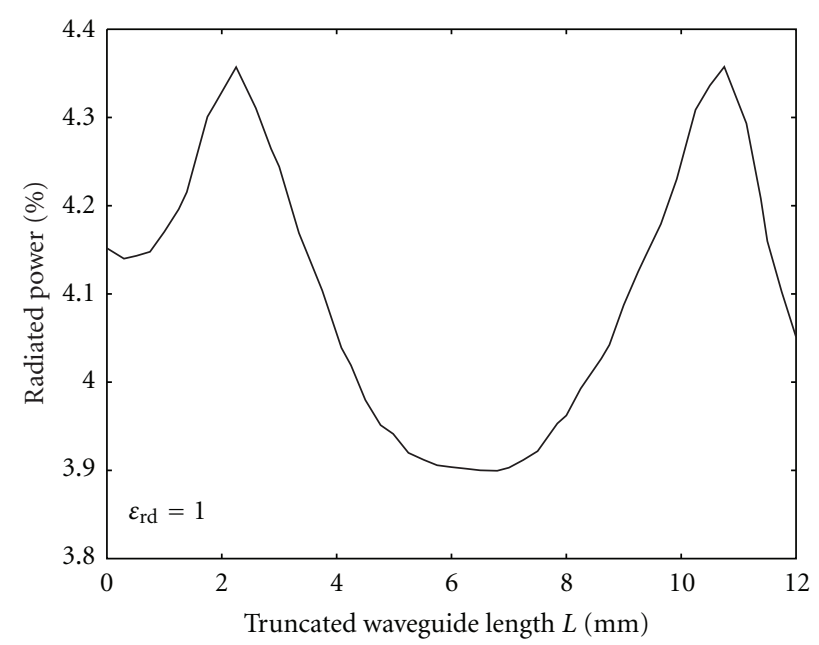

(a)

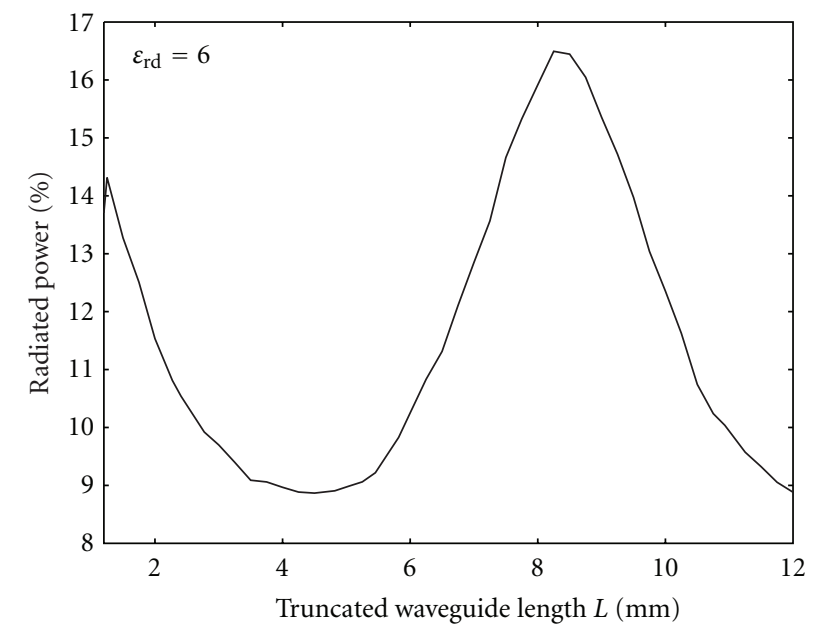

(b)

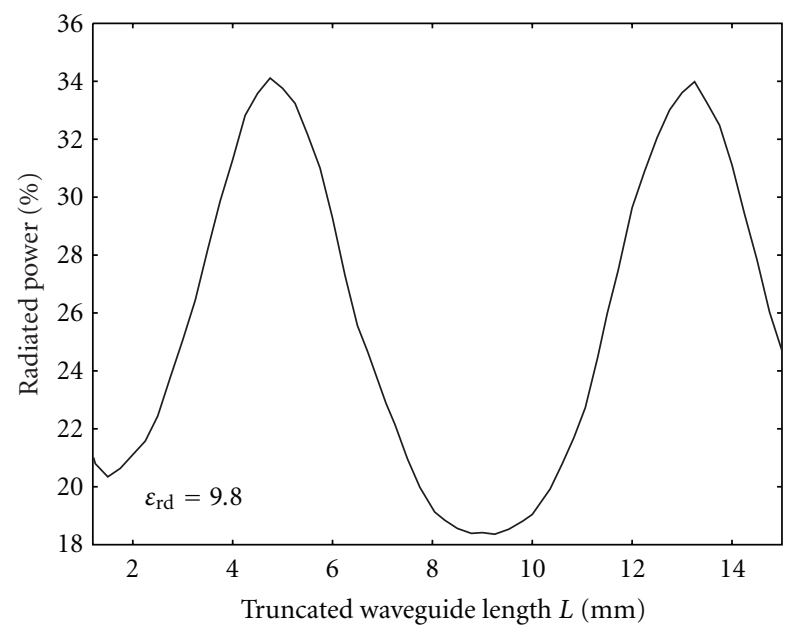

(c)

FIGURE 5: Radiated power (percentage of incident power) of the antenna in Figure 2. Radiating element parameters: $L_{S}=2.0 \mathrm{~mm}$, $W_{S}=0.3 \mathrm{~mm}$, and $h_{d}=1.2 \mathrm{~mm}$. 


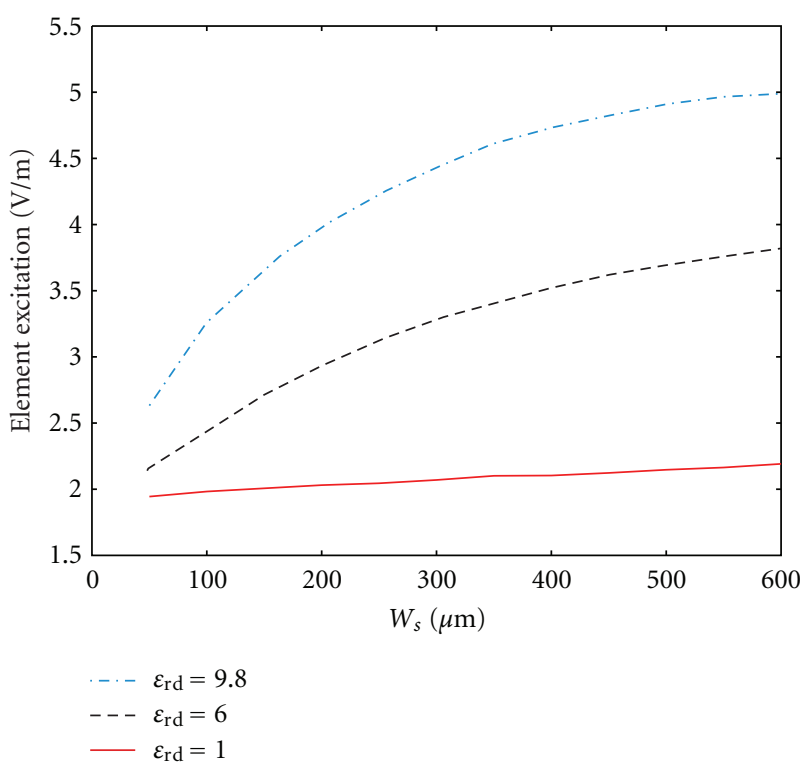

(a)

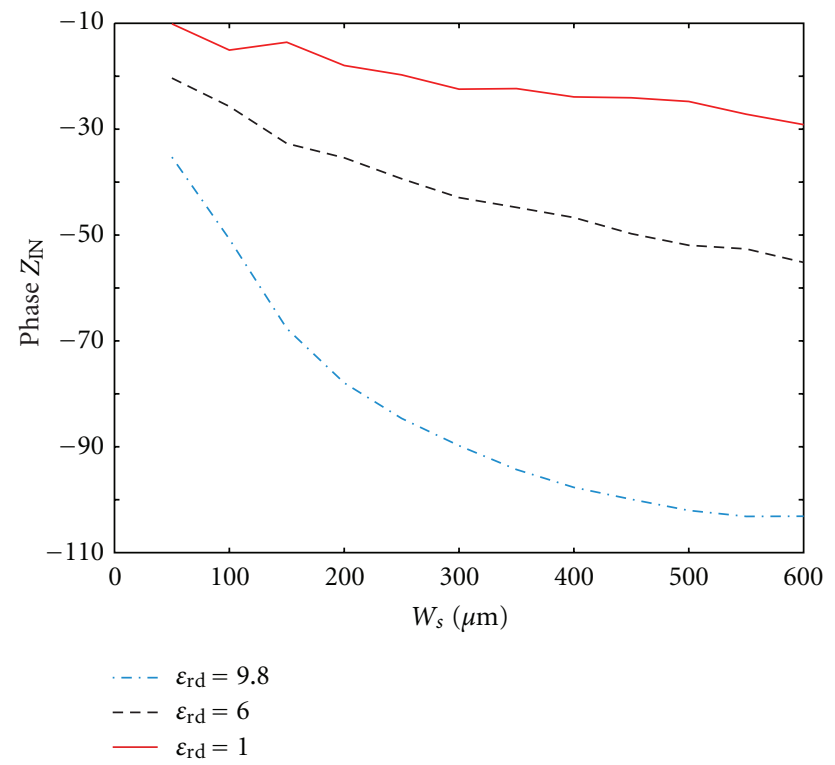

(b)

FIGURE 6: Element excitation (a) and phase of the input impedance (b) versus $W_{S}$ for different values of the dielectric permittivity $\varepsilon_{\text {rd }}$ (see Figure 2), $L_{S}=2 \mathrm{~mm}$, and $h_{d}=1.2 \mathrm{~mm}$.

filled with a dielectric substrate with $\varepsilon_{\text {rd }}=9.8$ and thickness $h_{d}=1.2 \mathrm{~mm}$.

The complete designed array has been analyzed using HFSS, since it has been shown that the results of this FEM software are in very good agreement with experiment (see e.g., [27]). In Figure 7, the radiation pattern at the center frequency of $24 \mathrm{GHz}$ is shown. The cross-polar component is below $-35 \mathrm{~dB}$, and the back-radiation level of the array is below $-25 \mathrm{~dB}$. In Figure $8(\mathrm{a})$, the co-polar far-field pattern is shown in the frequency band $23.8-24.2 \mathrm{GHz}$ and, in Figure $8(\mathrm{~b})$, the array gain is reported. The envelope of the

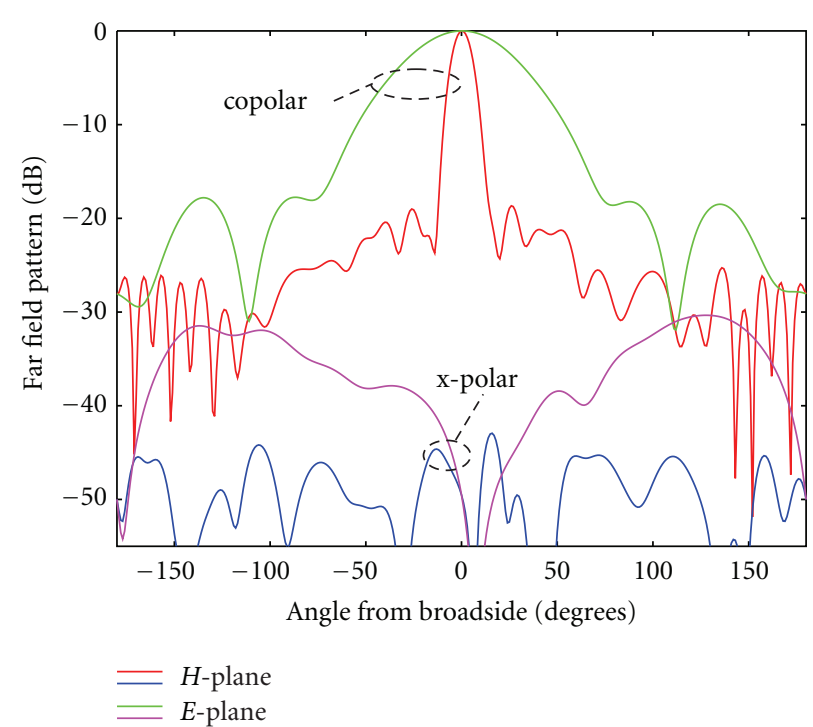

FIGURE 7: Radiation pattern of the 8-element array at the design frequency (HFSS data).

TABLE 1: Widths and excitations of the slots for the designed array.

\begin{tabular}{lccc}
\hline Element number & $\begin{array}{c}W_{S} \\
(\mathrm{~mm})\end{array}$ & $\begin{array}{c}\text { Excitation } \\
(\mathrm{V} / \mathrm{m})\end{array}$ & Normalized excitation \\
\hline 1,8 & 0.15 & 3.70 & 0.746 \\
2,7 & 0.20 & 3.97 & 0.800 \\
3,6 & 0.35 & 4.72 & 0.952 \\
4,5 & 0.55 & 4.96 & 1.000 \\
\hline
\end{tabular}

SLL is below $-17 \mathrm{~dB}$ in the whole frequency band, therefore, the far-field behavior is satisfactory in a frequency range of $400 \mathrm{MHz}$.

The finite extension of both the microstrip ground plane and truncated waveguide ground plane (see Figure 2(a)) has been taken into account in the HFSS simulation and we have found that an extension of $25 \mathrm{~mm} \times 100 \mathrm{~mm}$ is enough to avoid second-order effects in the array far-field pattern.

On the other hand, the array return loss in the frequency range is quite high, so a suitable matching network is needed. HFSS is able to compute the array input data over a large bandwidth, so that these data can be used both to match the array in its operational frequency range and to evaluate the out-of-band rejection.

The in-band input admittance $Y_{\mathrm{IN}}(\omega)$ is shown in Figure 9.

It appears that $Y_{\mathrm{IN}}(\omega)$ is the admittance of a RLC parallel resonant circuit, at least in the range $23.8-24.2 \mathrm{GHz}$. The circuital parameters can be easily evaluated by a linear fit and are $R_{E Q}=18 \Omega, C_{E Q}=58.137 \mathrm{pF}$, and $L_{E Q}=0.7461 \mathrm{pH}$. This RLC circuit is strongly resonant, with a resonant frequency of $24.15 \mathrm{GHz}$. This shift in the resonant frequency is due to the mutual coupling, neglected in the design. However, since this RLC circuit has a $Q=160$, the matching circuit must be designed starting from a BPF model [21], and this allows also to center the operating frequency range. 


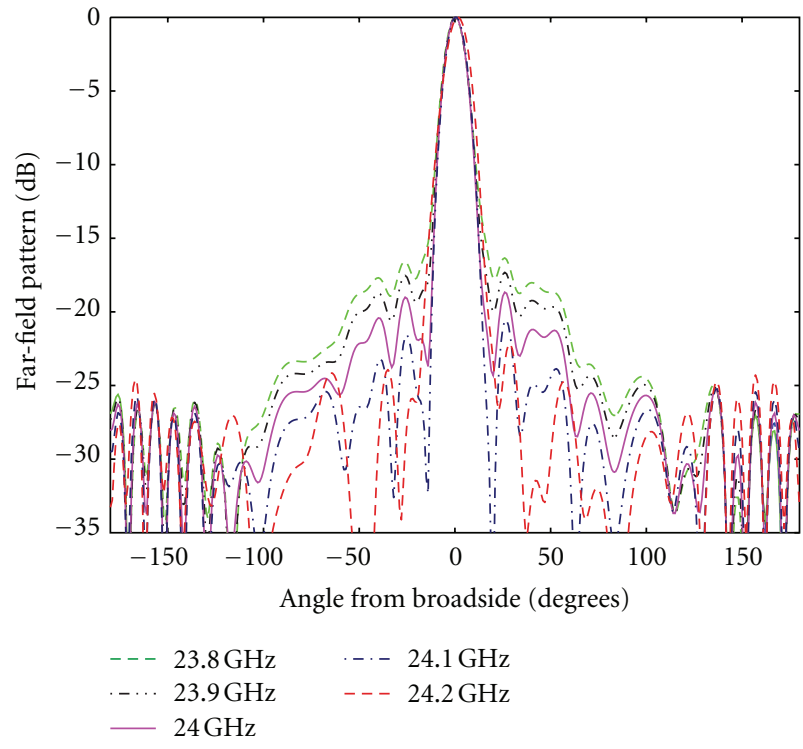

(a)

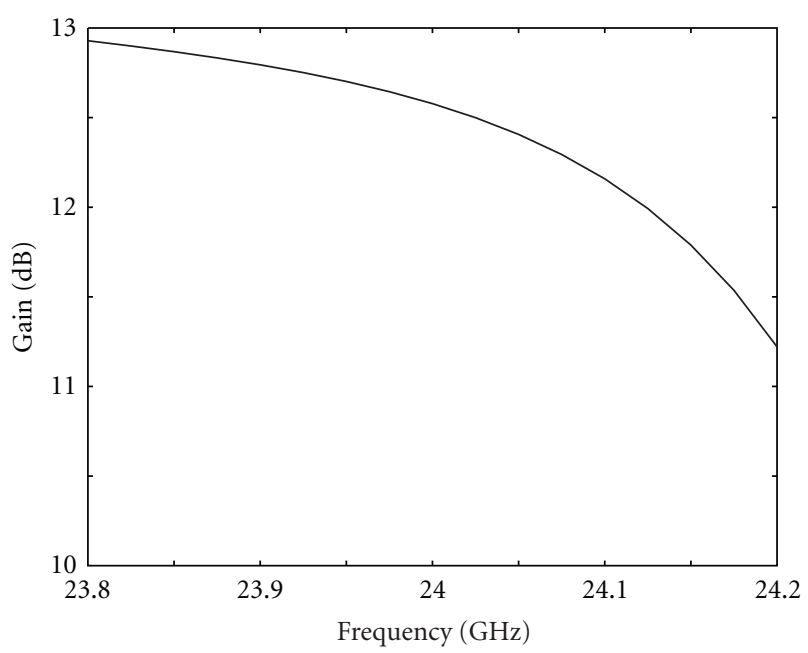

(b)

FIGURE 8: (a) $H$-plane radiation pattern for the 8-element array (HFSS data). (b) Array gain.

The large value of the load $Q$ calls for a BPF implementation using capacitive coupled resonators with a $Q$ significantly higher than load $Q$. Therefore, we have used a shielded microstrip line [28] for the filter, using the same PCB of the array, and with a total box dimension equal to $25 \mathrm{~mm} \times 1.381 \mathrm{~mm}$.

The final matching network is shown in Figure 10. $L_{T}=$ $19.894 \mathrm{pH}$ is required to center the resonant frequency at $24 \mathrm{GHz}$, while the capacitors are equal to $C_{1}=14.589 \mathrm{fF}$, $C_{2}=3.581 \mathrm{fF}$, and $C_{3}=8.355 \mathrm{fF}$ and were chosen to realize a 3 -pole Chebychev response.

Note that, since the input line is $50 \Omega$ and the output one is $35 \Omega$, one of the line resonators must be split into two parts. We choose the second one, with a total length of $2 \lambda$ to use a $C_{2}$ capacitor large enough to be realized. Moreover, we need

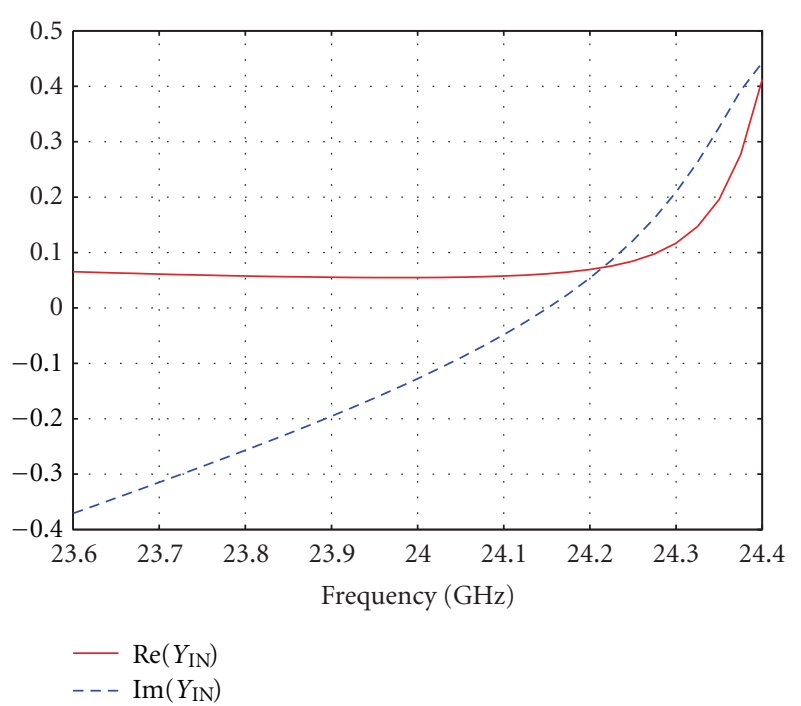

Figure 9: Input Admittance of the 8-element array (HFSS data), normalized to $0.02 \mathrm{~S}$.

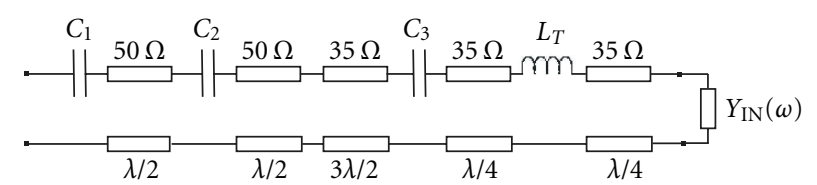

Figure 10: Transmission Line Model of the matching network.

a further line of $35 \Omega$ at the end to accommodate, with the smallest increase of the load $Q$, the slit inductor [22].

The final layout is shown in Figure 11. The capacitor $C_{2}$ has been split into two capacitors $2 C_{2}$ in order to avoid a too large gap. The array return loss is reported in Figure 12(a) and the operating bandwidth is around $210 \mathrm{MHz}$. In Figure 12(b), the same frequency response is computed over a large bandwidth to evaluate the out-ofband rejection. It appears that over a $12 \mathrm{GHz}$ frequency range, the antenna presents a reactive load (except, of course, in its operational bandwidth). Therefore, when used as a receiving antenna, the first band-pass filter is not required [29].

\section{Conclusion}

In this paper, a new high-performance antenna for application in automotive anti-collision radars has been presented. The proposed antenna allows to obtain a high radiated power and presents a very low cross-polar component and back radiation. A well-assessed MoM code has been used to analyze this radiating element, modeling the microstrip feeding line by its equivalent magnetic-wall waveguide.

A linear array, suitable for anti-collision radar, has been designed and then evaluated by using HFSS. A matching network, designed for a $200 \mathrm{MHz}$ bandwidth around $24 \mathrm{GHz}$, allows an in-band behavior suitable for radar use, and guarantees a high out-of-band rejection. 


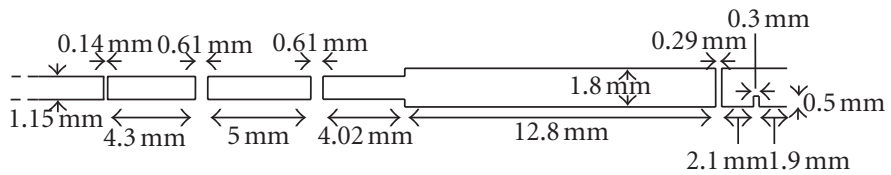

Figure 11: Layout of the matching network.

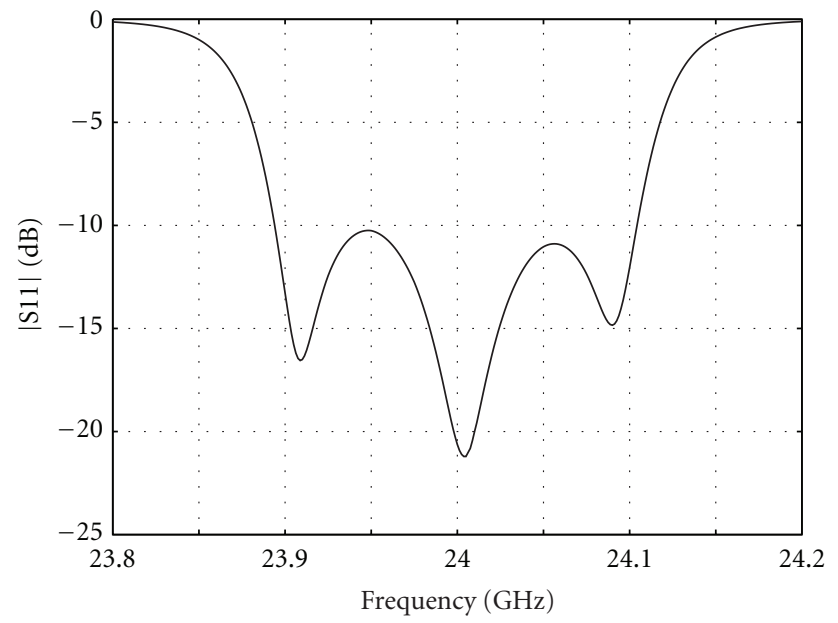

(a)

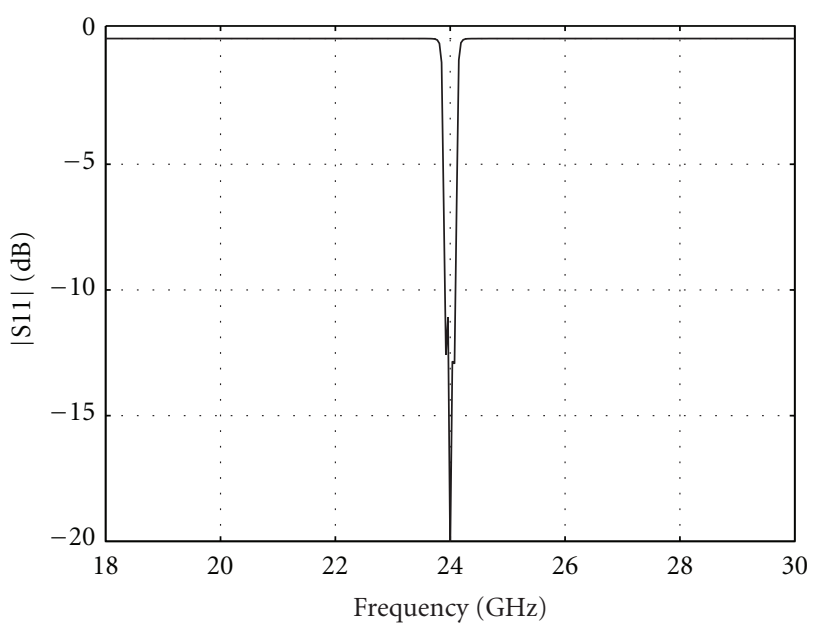

(b)

FIGURE 12: (a) In-band return loss of the matched array. (b) Outof-band return loss of the matched array.

\section{References}

[1] P. L. Lowbridge, "Low cost millimeter-wave radar systems for intelligent vehicle cruise control applications," Microwave Journal, vol. 38, pp. 20-33, 1995.

[2] L. H. Eriksson and B. O. As, "High performance automotive radar for automatic AICC," IEEE Aerospace and Electronic Systems Magazine, vol. 10, no. 12, pp. 13-18, 1995.

[3] F. Folster, H. Rohling, and U. Lubbert, "An automotive radar network based on $77 \mathrm{GHz}$ FMCW sensors," in Proceedings of the IEEE International Radar Conference, pp. 871-876, May 2005.
[4] M. M. Balas, V. E. Balas, and J. Duplaix, "Optimizing the distance-gap between cars by constant time to collision planning," in Proceedings of the IEEE International Symposium on Industrial Electronics (ISIE '07), pp. 304-309, June 2007.

[5] ElectroMagnetic Compatibility and Radio Spectrum Matters (ERM), Road Transport and Traffic Telematics (RTTT), and Traffic Telematics (RTTT), "Technical characteristics and test methods for radar equipment operating in the $76 \mathrm{GHz}$ to $77 \mathrm{GHz}$ band. Part 1: technical characteristics and test methods," ETSI EN 301 091-1.

[6] X. Feng, C. Xu, and W. Xin'an, "K-band micro-strip antenna array applied in anti-collision radar," in Proceedings of the IEEE 12th International Conference on Communication Technology (ICCT'10), pp. 1240-1243, November 2010.

[7] L. Moon-Sik and K. Yong-Hoon, "Design and performance of a 24-GHz switch-antenna array FMCW radar system for automotive applications," IEEE Transactions on Vehicular Technology, vol. 5, pp. 2290-2297, 2010.

[8] C. Wang, K. Qian, M. H. Yang, Y. Sun, J. Z. Gu, and X. W. Sun, "A low cost 24-GHz FMCW radar for automobile application," in Proceedings of the 2nd European Radar Conference (EURAD '05), pp. 375-378, October 2005.

[9] M. Slović, B. Jokanović, and B. Kolundžija, "High efficiency patch antenna for $24 \mathrm{GHz}$ anticollision radar," in Proceedings of the 7th International Conference on Telecommunications in Moderm Satellite, Cable and Broadcasting Services (TELSIKS '05), pp. 20-23, September 2005.

[10] S. H. Jeong, H. Y. Yu, J. E. Lee, J. N. Oh, and K. H. Lee, "A multi-beam and multi-range radar with fmcw and digital beam forming for automotive applications," Progress In Electromagnetics Research, vol. 124, pp. 285-299, 2012.

[11] D. M. Pozar, Microstrip Antennas: The Analysis and Design of Microstrip Antennas and Arrays, IEEE Press, New York, NY, USA, 1995.

[12] M. Himdi, J. P. Daniel, and C. Terret, "Analysis of aperturecoupled microstrip antenna using cavity method," Electronics Letters, vol. 25, no. 6, pp. 391-392, 1989.

[13] G. Montisci, G. Mazzarella, and G. A. Casula, "Effective analysis of a waveguide longitudinal slot with cavity," IEEE Transactions on Antennas and Propagation, vol. 60, pp. 31043110, 2012.

[14] I. Wolff, The Waveguide Model for the Analysis of Microstrip Discontinuities, Numerical Technique for Microwave and Millimeter-Wave Passive Structures, Edited by Itoh T., WileyInterscience, NewYork, NY, USA, 1989.

[15] G. A. Casula, G. Mazzarella, and G. Montisci, "Effective analysis of a microstrip slot coupler," Journal of Electromagnetic Waves and Applications, vol. 18, no. 9, pp. 1203-1217, 2004.

[16] G. Mazzarella and G. Montisci, "Accurate Characterization of the Interaction between Coupling Slots and Waveguide Bends in Waveguide Slot Arrays," IEEE Transactions on Microwave Theory and Techniques, vol. 48, no. 7, pp. 1154-1157, 2000.

[17] S. Costanzo, G. A. Casula, A. Borgia et al., "Synthesis of slot arrays on integrated waveguides," IEEE Antennas and Wireless Propagation Letters, vol. 9, pp. 962-965, 2010. 
[18] G. Mazzarella and G. Montisci, "Accurate modeling of coupling junctions in dielectric covered waveguide slot arrays," Progress In Electromagnetics Research M, vol. 17, pp. 59-71, 2011.

[19] S. Costanzo, I. Venneri, G. Di Massa, and G. Arriendola, "Hybrid array antenna for broadband millimeter-wave applications," Progress in Electromagnetics Research, vol. 83, pp. 173-183, 2008.

[20] http://www.ansys.com.

[21] H. Kanaya, M. Kato, D. Kanemoto, K. Yoshida, R. K. Pokharel, and K. Yoshitomi, "Development of $2.4 \mathrm{GHz}$ onesided directional slot antenna with 2-stage bandpass filter," in IEEE Antennas and Propagation Symposium, 2012.

[22] Z. Wang, P. S. Hall, and P. Gardner, "Yagi antenna with frequency domain filtering performance," in IEEE Antennas and Propagation Symposium, 2012.

[23] S. R. Rengarajan, "Compound radiating slots in a broad wall of a rectangular waveguide," IEEE Transactions on Antennas and Propagation, vol. 37, no. 9, pp. 1116-1123, 1989.

[24] Q. Rao, T. A. Denidni, and R. H. Johnston, "A singlesubstrate microstrip-fed slot antenna array with reduced back radiation," IEEE Antennas and Wireless Propagation Letters, vol. 3, no. 1, pp. 265-268, 2004.

[25] Q. Rao, T. A. Denidni, and R. H. Johnston, "Dielectric reflector backed aperture-coupled antennas for reduced back radiation," IEEE Transactions on Electromagnetic Compatibility, vol. 48, no. 2, pp. 287-291, 2006.

[26] M. Wang, W. Wu, and Z. Shen, "Bandwidth enhancement of antenna arrays utilizing mutual coupling between antenna elements," International Journal of Antennas and Propagation, vol. 2010, Article ID 690713, 9 pages, 2010.

[27] S. R. Rengarajan, M. S. Zawadzki, and R. E. Hodges, "Waveguide-slot array antenna designs for low-average-sidelobe specifications," IEEE Antennas and Propagation Magazine, vol. 52, no. 6, pp. 89-98, 2010.

[28] B. C. Wadell, Transmission Line Desing Handbook, Artech House, Norwood, NJ, USA, 1991.

[29] Z. Wang, P. S. Hall, P. Gardner, and J. H. Wu, "Yagi antenna with improved out-of-band gain suppression," Electronics Letters, vol. 48, pp. 546-548, 2012. 

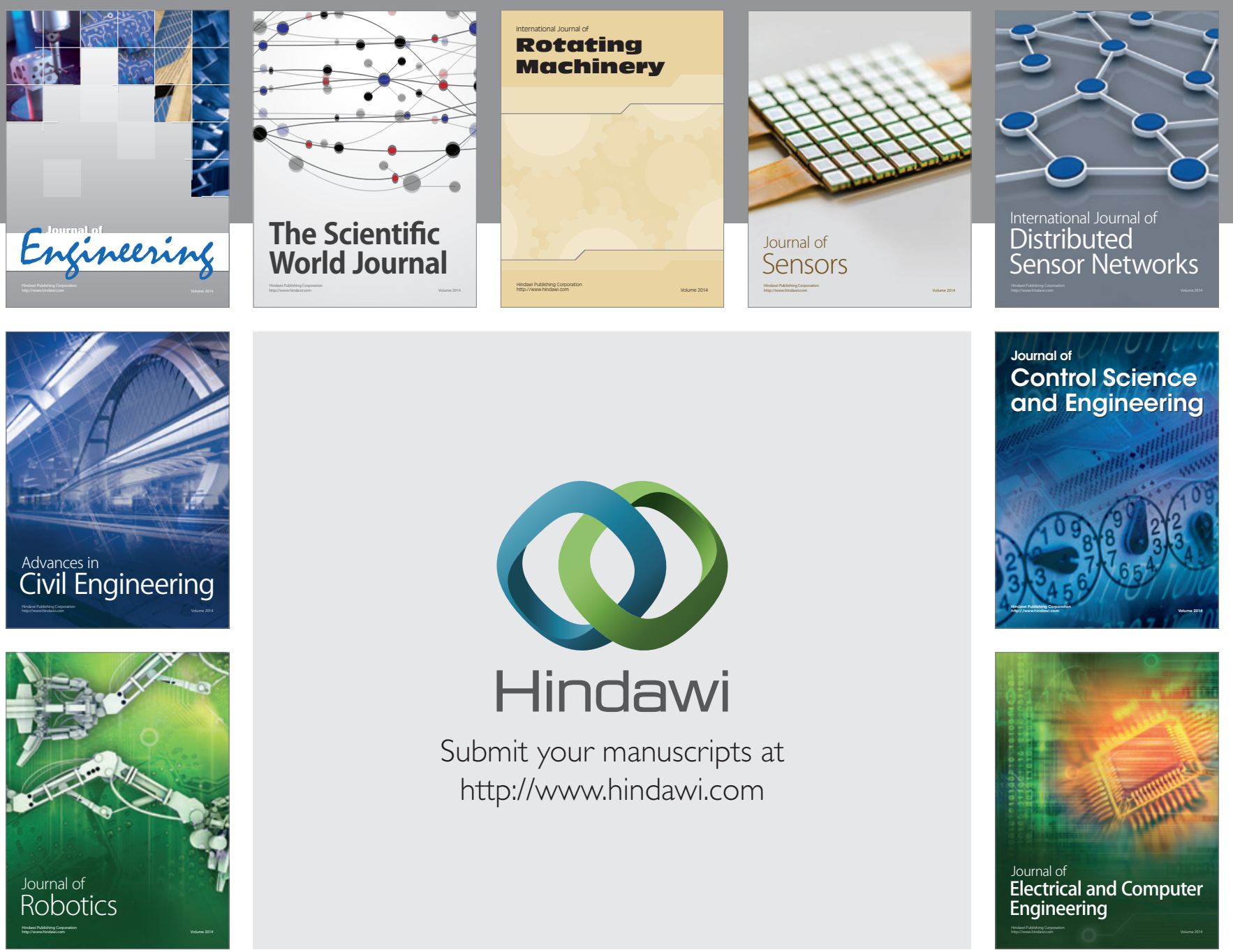

Submit your manuscripts at

http://www.hindawi.com
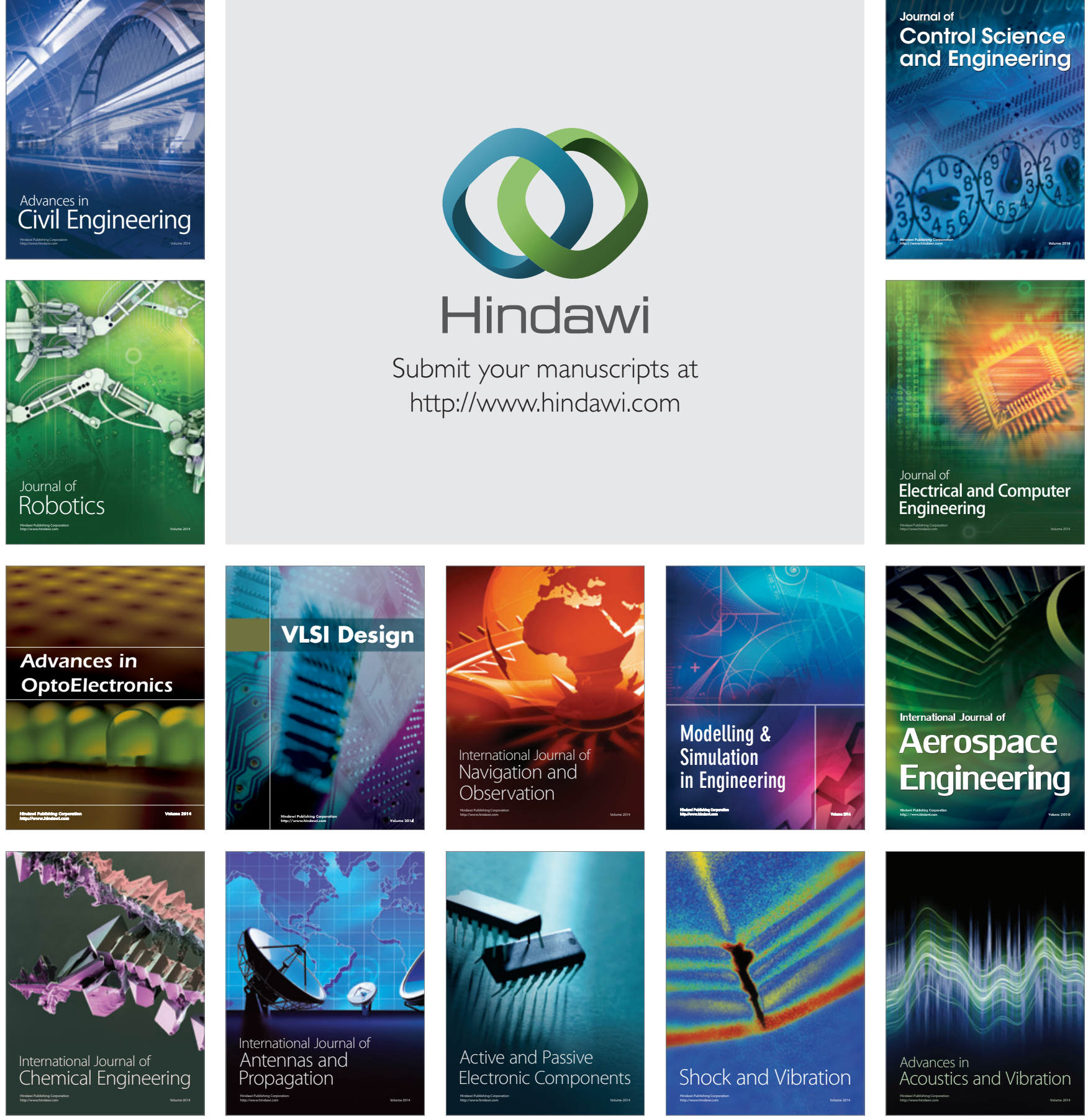